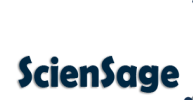

Journal of Advanced Scientific Research

Available online through https: / /sciensage.info
ISSN: 0976-9595

Review Article

DOI: $10.55218 /$ JASR. 202213102

\title{
SEVERITY OF COVID-19 IN INDIAN CHILDREN: RECOGNITION, TRANSMISSION AND MANAGEMENT
}

\author{
Sanjukta Manna*, Esha Basu, Satrajit Das \\ Post-Graduate Department of Zoology, Maulana Azad College, 8 Rafi Ahmed Kidwai Road, Kolkata, West Bengal, India \\ *Corresponding author: sanjukta_manna@rediffmail.com
}

\begin{abstract}
Coronavirus disease (COVID-19) caused by Severe Acute Respiratory Syndrome Corona Virus 2 (SARS-CoV2) has become a global threat in recent times affecting millions of people across the globe with varying intensity of infections in different age groups. The disease has been reported with a considerably low infection rate among the children compared to that of adults to date, but the reasons are still not very clear to the scientists. As infants are naturally prone to a wide range of viruses, it is fascinating to understand that they are being least affected by SARS-CoV-2. Expression of Angiotensin Converting Enzyme 2 (ACE2) and Transmembrane Serine Protease (TMPRSS2) Receptors have been thought to play a critical role in the expression of the disease. The shared genetic or environmental traits and socioeconomic condition might predispose for transmission dynamics of this coronavirus in children. The children genetic association may have risk of small amount although genetic variants of SARS-CoV-2 are comparable to those for the risk factors such as obesity, diabetes and other underlying health conditions. Here in this review, we have focused on the differential expression of SARS-CoV-2, the severity of the disease, predisposing factors, preventive measures, and efficacy of vaccines among the different age groups of Indian children in a comprehensive manner.
\end{abstract}

Keywords: COVID-19, SARS-CoV-2, ACE2, TMPRSS2, Infants, Multisystem inflammatory syndrome.

\section{INTRODUCTION}

The global pandemic of coronavirus disease-2019 (COVID-19) [1] caused by the novel severe acute respiratory syndrome coronavirus-2 (SARS-CoV2) triggered urgent research on the pathogenesis and transmission to children and adolescents. Coronaviruses are a group of viruses that belong to the Coronaviridae family. Coronaviridae is a family of enveloped, singlestranded, positive-sense RNA virus and can be divided into four genera: $\operatorname{alpha}(\alpha), \operatorname{beta}(\beta), \operatorname{gamma}(\gamma)$, and $\operatorname{delta}(\delta)[2]$. Different variants of COVID 19 evolve through mutations of spike protein and as a result, their characteristics undergo rapid changes like community transmission and the severity of the disease. Recently, a new virulent version of the highly contagious delta variant (B.1.617.2) has emerged as a new threat especially in India named AY.1 or the delta plus. Mutation in the delta variant led to the formation of delta plus strain. The new strain carries K417N mutation in the spike protein which enables the virus to bind more tightly. The concerning new variant possesses two worrying features: higher transmission rate and monoclonal antibody resistance. 56 cases with delta variant have been detected in the genomes sequenced so far [3] in India. Children at all ages were sensitive to COVID-19 but severity varies from asymptomatic to lethal condition and there was no significant gender difference (Fig1). The distribution of children's COVID-19 cases in India varied with time, climate and space, and most of the cases concentrated in metro cities. The currently ongoing COVID-19 pandemic has involved more than 190 countries and children are affected less frequently [4].

Children are the heart of the fears around a possible third wave of COVID-19 in India. Asymptomatic transient nasopharyngeal colonization with Corona virus tends to be most severe in infants $<1$ year of age and in children with underlying health problems. In most children, COVID 19 causes no or only mild symptoms. Infected children might appear with fever, dry cough, fatigue and few have upper respiratory symptoms including nasal congestion and running nose. Some children present with gastrointestinal symptoms including abdominal discomfort, nausea, vomiting and diarrhoea. Many literatures on COVID-19 paid attention to the long-term effects of COVID-19 and the 
commonly used term for this is long COVID. A small proportion of children develop hyperinflammation which is described as multi-inflammatory syndrome in children (MIS-C) [5]. A rare inflammatory and potentially life-threatening condition called multisystem inflammatory syndrome (MIS-C)usually develops four to six weeks after children and teenagers have recovered from COVID-19 as result of enhanced immune response to the severe acute respiratory syndrome coronavirus 2 (SARS-CoV-2), which can lead to inflammation of vital organs. Symptoms are high and persistent fever, rash, red eyes, inflamed lymph nodes, stomach pain, low blood pressure, body ache and lethargy.

While data are scarce, we tried to analyse the necessity of high vigilance among primary care and emergency doctors, and preparedness during the COVID - 19 pandemic in India with a high proportion of malnourished and immunocompromised children.

\section{CHANCES OF SEVERITY OF THE DISEASE}

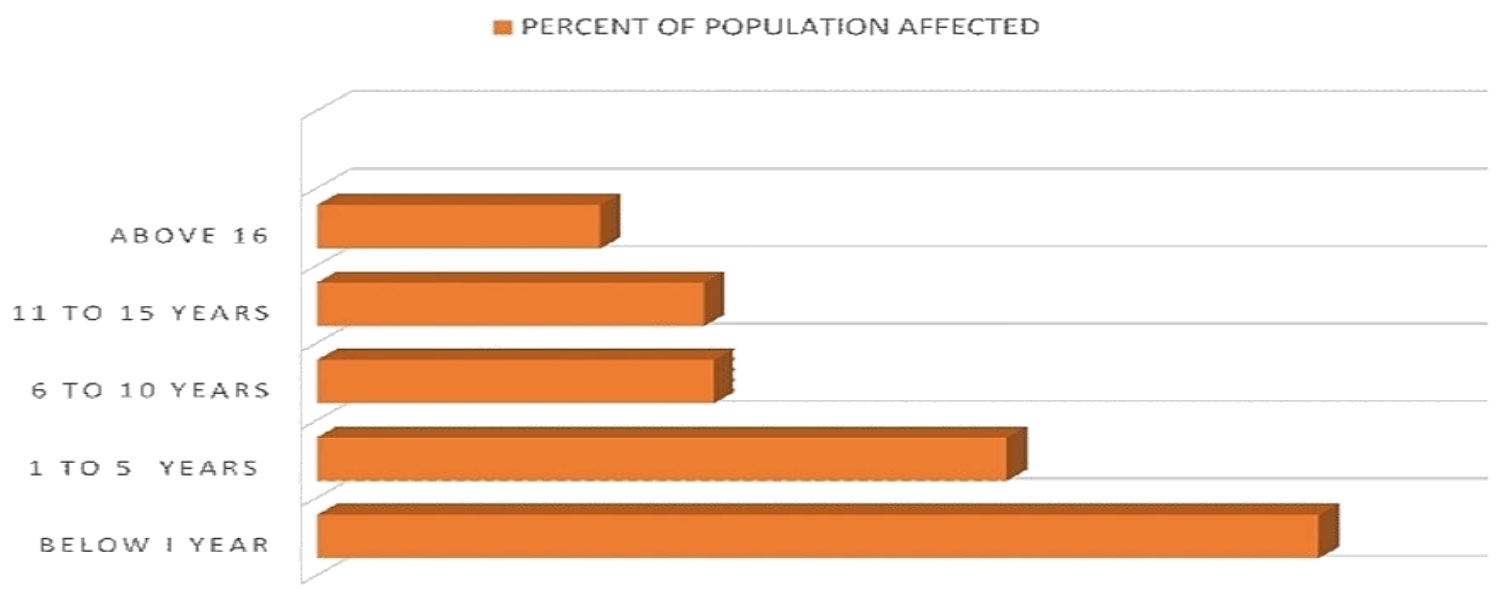

Fig. 1: Severity of the disease among children (in different age groups)

\section{WHY THE CHILDREN HAVE BEEN LEAST AFFECTED TO DATE}

Several probable reasons have been suggested for the transmission and impact of the disease by a different group of scientists. But the cases among the children have been considerably low to date. Different case studies have shown that the majority of children who have been infected with the virus are asymptomatic and are completely fine but a few portions of the symptomatic individuals had to be admitted into the hospital, out of them a lesser number were to be supported with the Intensive Care Units (ICU). Taking all these together, the number of individuals below 12 years of age admitted to the ICU is negligible. But case studies revealed that the children with complexities with this virus with comorbidities are at a higher risk. The major symptoms of the children includeinflammations in upper respiratory (cold and cough, running nose, etc.), and lower respiratory tracts (like Asthma, shortness of breath) and seldom occurrence of respiratory distress syndrome (ARDS) which includes cardiac arrest leading to septic shock and ultimately leads to the death. Cytokine storm is very rare to be seen among children.

\subsection{Differential immune response of child compared to adult}

Differences in immunity between children and adults have also been believed to a major cause of the majority of children being asymptomatic or showing mild symptoms of the disease. Even the infants of different ages like very young children, pre-school children, and teenagers also show a differential immune response. Some of the maternal antibodies also do persist since very early childhood, giving more protection towards the antigens.

\subsection{Virus-virus interactions}

The young children have also the advantages that the mucosa of their upper respiratory tract naturally harbours several viruses including common cold, influenza, etc. One possible theory is that the SARSCoV-2 has to compete with them to get the space to infect host cell overcoming a virus-virus interaction [6]. 


\subsection{Number of Angiotensin Converting Enzyme2} (ACE2) and Transmembrane serine protease (TMPRSS2) receptors

Considering the respiratory and olfactory epithelium in the nasal mucosa as one of the first contact points for the Corona virus, ACE2 expression in nasal epithelium may have an important role in SARS-CoV-2 infection and transmission. The number of ACE2 receptors is remarkably lower in the upper and lower respiratory tract of infants compared to that of adults. As ACE2 receptors are necessary for the attachment and downregulation of the SARS-CoV-2 by receptormediated endocytosis, the organs showing ACE2 receptors on their membrane are the major targets of the Coronavirus including the heart, lungs, kidneys, and gastrointestinal tract [7]. The TRPSS2 receptor being organ-specific, though it has a role on the proteolytic cleavage of the SARS-CoV-2 spike glycoprotein to mediate its entry into the host cell [Fig2], it is not expressed in all types of organs [8]. SARS-CoV-2 in humans has a 10-20 fold higher affinity to ACE2 receptors than other respiratory viruses [9] as respiratory binding domain of spike glycoprotein binds to ACE2. The receptor binding domain found on S1 subunit of $S$ protein is vital for the binding of ACE2 to the virus. Case studies have proved that the patients with comorbidities, COPD, asthma, hypertension, and diabetes have a considerably elevated number of ACE2 receptors in the upper and lower respiratory tracts including some other organs [10]. The majority of children are not concerned with these physiological complexities and show a lower number of ACE2 receptors which is a possible cause of the mild symptoms or the majority of the children being asymptomatic [4]. Hasan and Sharma, [11] observed that children have lower transcript levels of nasopharyngeal ACE2 and TMPRSS2, which have important prognostic implications against infection from SARS-CoV-2 when exposed to COVID-19 positive adult family members.

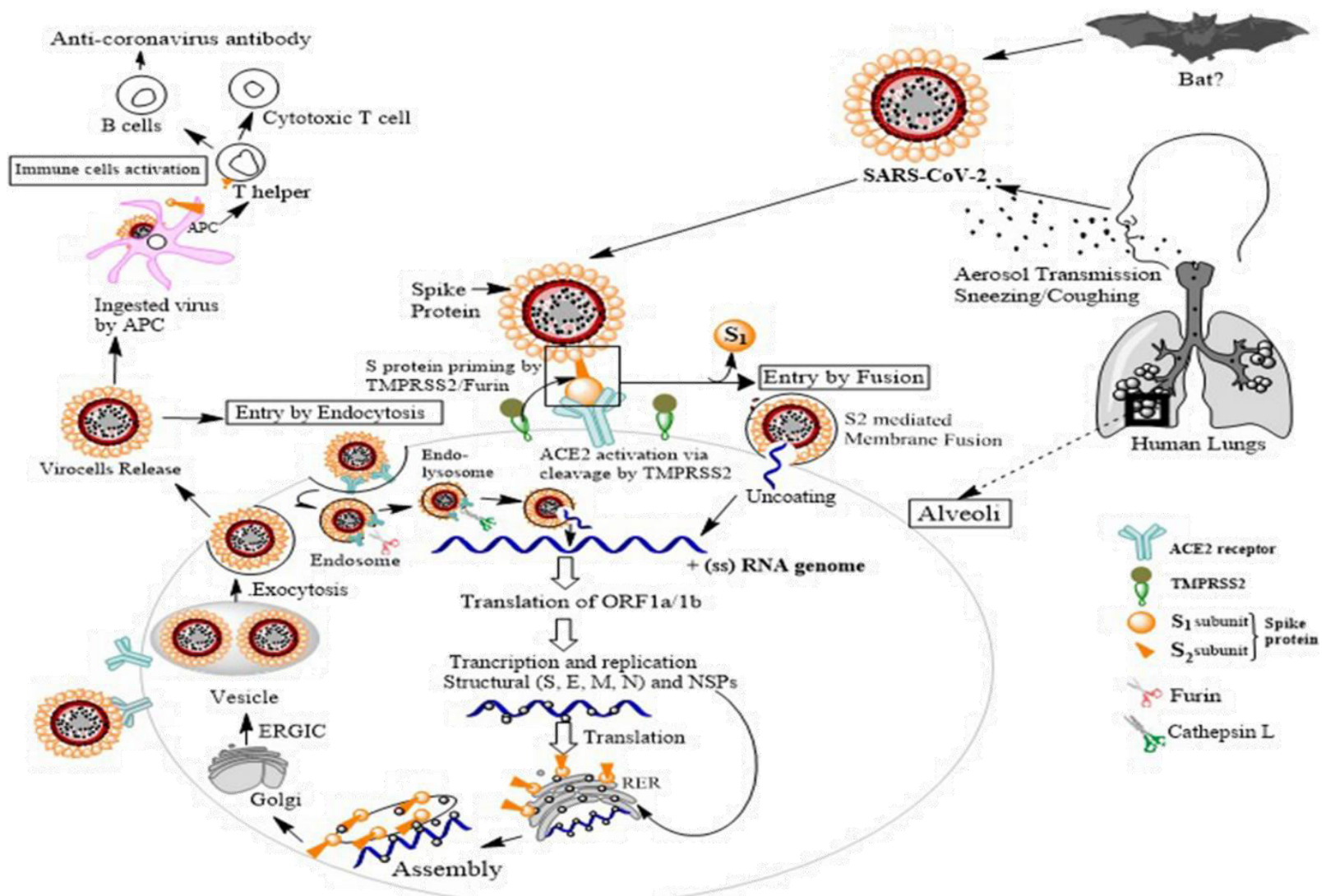

SARS-Cov-2 binds to ACE2 receptor present in alveolar cells of human lungs and fuses with membrane,this requires the two domains S1 and S2 of spike protein to be cleaved using TMPRSS2.+ ssRNA genome translates two ORFs (1a and $1 b$ ) which can further transcribe and replicate into structural(S,M,E,N) and nonstructural proteins(NSPs). The virion proteins are processed through rough endoplasmic reticulum and Golgi complex. The virocells are released through exocytosis by endoplasmic reticulum Golgi intermediate compartment (ERGIC). The viral S peptide presents to Thelper cells through APC.

Fig. 2: The cellular entry and replication mechanism of SARS-Cov-2 in human cell. 
According to the Union Government, 90 per cent of the Covid cases in India during the deadly second wave of the Covid-19 pandemic in April and May, are being driven by the B16172 (Delta: three sub-lineages, namely B.1.617.1, B.1.617.2, and B.1.617.3.) variant. The spike protein of delta variant contains nine mutations in the $\mathrm{S} 1$ subunit and one mutation in the S2 subunit. In the S1 subunit, five mutations are present in the N-terminal domain containing binding sites (epitopes) for neutralizing antibodies. In addition, two mutations are present in the receptor-binding domain of the $\mathrm{S} 1$ subunit, which is known to influence antibodymediated neutralization and infectivity. Among the three remaining mutations, two are known to increase angiotensin-converting enzyme 2 (ACE2) binding, viral replication, and spike protein cleavage at the S1/S2 site [12].

India's health ministry says studies showed that the socalled delta plus variant (Delta variant with an additional mutation, also known as $\mathrm{K} 417 \mathrm{~N}$ and AY.1 lineage (B.1.617.2.1) (INSACOG,2021) spreads and binds more easily to lung cells and is potentially resistant to monoclonal antibody therapy, a potent intravenous infusion of antibodies to neutralise the virus. The delta plus new variant of COVID-19, which has created fresh concerns across the country, cannot be yet linked to a third wave of the pandemic, scientists of IIT Kanpur has confirmed that the third wave of COVID-19 is expected by September to October 2021. Indian Children are affected by COVID-19, but from extant data children are less likely to be affected than adults [13-15].

The Report of the WHO-China Joint Mission on COVID-19 [16] stated that "We cannot determine the extent of infection among children, what role children play in transmission, whether children are less susceptible or whether they simply present differently."

\subsection{Nutrition}

The COVID 19 pandemic increases social and economic crisis globally that poses grave risks to the nutritional status and survival of young children in low-income and middle-income countries. 2020 Global Nutrition Report have stated, "Malnutrition is a threat-multiplier. If no action is taken, the effects of the current COVID19 pandemic will only make it harder for vulnerable populations to protect themselves against malnutrition." Urban poverty makes the Indian children nutrient deficient. Iron, iodine, folate, vitamin A, and zinc deficiencies invite many viral diseases like COVID 19, swine flu and fungal disease like mucormycosis. Vitamin
D may play a special role in reducing the risk of respiratory tract infections, with a potential for lessening the effect of COVID-19. Vitamin D increases the production of natural antibodies [17] and strengthens the immunity by inducing monocyte differentiation and inhibiting lymphocyte proliferation [18]. It also postulated that vitamin D enhances the phagocytic activity of macrophages.

Nutrition is intrinsically linked to the immune system and to disease susceptibility. In this situation financial assistance to poor families is crucial that provide the affordability of healthy diets with vitamin supplements who remain out of reach for numbers of families.

\subsection{Comorbidities}

The children with underlying conditions are at greater risk of infection or more severe disease, although the evidence is limited and mixed. Children with a preexisting major disease mostly showed severity after getting infected with corona virus for example, heart, kidney, liver disease, or malignancies.

Children with obesity, diabetes, asthma or chronic lung disease, sickle cell disease, or immunosuppression might also be at increased risk for severe illness from COVID-19. Children with nonsevere COVID-19 may require hospital admission if they are at risk for severe disease due to underlying conditions (eg, immune compromise). Although the relationship between immune compromise and severe COVID-19 disease has not been well established in children [19, 20], management of viral infections in immunocompromised hosts typically includes reduction of baseline immunosuppression.

\subsection{Genetic hits}

Genetic variants in human population usually have minimum effects on infection outcomes compared with the Risk factors like age, comorbidities and environmental factors including socio-economic determinants of healthare known to have roles in determining COVID 19 severity.

ACE2 and TMPRSS2 DNA polymorphisms have been shown to be strongly associated with the susceptibility, severity, and clinical outcomes of COVID-19 [21]. SARS-CoV-2 utilizes molecules to enter the cells, such as angiotensin-converting enzyme 2 (ACE2) to attach to the receptor-binding domain (RBD) and type 2 transmembrane serine proteases (TMPRSS2) to cleave the spike (S) protein $[22,23]$. SARS-CoV-2 S-protein 
cleavage by TMPRSS2 initiates viral entry and also helpsthe virus escape the immune system. The hidden receptor-binding domain attached to ACE2 enhances immune evasion by weakening the immune surveillance. SARS-CoV-2 S-protein cleavage by TMPRSS2 is also responsible for immune escape besides initiating the viral entry [22]. Hence, genetic differences among such molecules responsible for cellular entry might alter the observed responses among different individuals [21, 24]. Recent literature reported that not only genetic differences can cause particular races and populations to be more affected by COVID 19, but also, they might contribute to gender-specific differences [25]. Higher testosterone levels enhance TMPRSS2 levels and might cause higher disease susceptibility in males [26, 27]. To make matters even more important, the differences in severity observed in children compared to older adults may be in parts due to the different expressions of genetic components, such as TMPRSS2 [28].

A family of antiviral genes called OAS (oligo adenylate synthase) activates enzymes to cleave viral RNA, and leads to lower circulating levels of the OAS1 enzyme in the lungs increases risk of infection, hospitalization and critical illness. The coronaviruses evade this protection using proteins called PDEs, or phosphodiesterases, but SARS-CoV-2 doesn't make PDEs [29].

The chromosome 3 regions have several genes involved in immune signalling, lung biology and other plausible mechanisms. But it's not clear which of these genes explains the association with COVID-19 [30].

The DOCK2 variant, an immune gene with a role in interferon production, increased COVID-19 risk was relatively common in East Asians, but very rare in people of European, South Asian and African ancestry. For that emphasis should give on the importance of increasing diversity in host genetics of COVID-19 [31].

\section{SYMPTOMS AND TRANSMISSION AMONG CHILDREN}

The transmission of infection among the children has been reported mainly by droplets, or aerosols. The majority of the cases have with family history but the children were asymptomatic. Samples which include nasopharyngeal swab have shown higher viral load than saliva. Though the virus has been reported to have in the stool of affected children, faecal-oral transmission has not yet been well marked. Immunocompromised children with malignancies have shown quite promising results with mild to moderate severity of the disease [11].

\subsection{Severity vs susceptibility}

Children are affected mildly by covid-19 infection. For those who are being affected, the severity is comparatively higher in babies and infants and in cases the children comparatively elder than the other age categories severely affected, are presented with the multisystem inflammatory syndrome in children (MISC) which exhibit symptoms resembling Kawasaki' sdisease(a condition where the coronary arteries enlarge or form aneurysms), Toxic Shock Syndrome (is a rare condition caused by bacterial infection) and myocarditis. The occurrence of the syndrome is not very clear. This syndrome might result from a delayed hypersensitivity and abnormal immune response to the virus resulting in inflammation which damages the organs.

\subsection{Specific Features of MIS-C}

MIS-C includes allergies, distended hands, and feet, inflamed mucous membrane. Cardiopulmonary symptoms include low blood pressure, difficulty in breathing, light headedness and irregular heart beat. Gastrointestinal symptoms include diarrhoea, vomiting, abdominal pain, Respiratory symptoms are cough, sore throat and shortness of breath, Neurological and cognitive symptoms include headache, seizure and confusion [10]. These were the discomforts observed in children and elevated levels of C-reactive protein, ddimer and troponin were found in $100 \%$. Almost all the children so far reported in India completely recovered, although rare deaths resulted from complications of extracorporeal membrane oxygenation. It has also been noticed that chloroquine and azithromycin, medicines used for treating covid-19, are affecting neuromuscular junction transmission and should be prescribed in children cautiously. Sometimes, medications like Lopinavir or Ritonavir for treating COVID 19 are causing confusion, drowsiness and ultimately multisystem inflammatory syndrome in children [4].

\subsection{Mental health of the children}

Prolonged lockdown has adversely affected a slew of people including adults and children. The effect on the mental health of children is multifaceted including a wide range of symptoms and susceptible to many communicable infections. Being physically detached from friends, school, relatives and several occasions have put on enormous mental pressure on the child to adolescents. These are giving rise to different kinds of anxiety and stress-related disorders in children and 
adolescents [32] . In this situation, adrenal insufficiency may put the children at higher risk of infections of Covid 19 due to a lack of normal stress response by the body. Adrenal insufficiency is a condition in which the adrenal gland does not secrete enough cortisol hormone. Endocrine Society of India suggested that it was important to take extra precautions to prevent and treat Covid-19 infection by cortisol replacement therapy in children with adrenal insufficiency [15].

\section{PREVENTIVE MEASURES}

\subsection{Vaccines}

As the vaccines have been considered as the most powerful preventive measure to combat COVID19, roll out the vaccines among the children should be started without further delay once the clinical trial is satisfactorily being completed. Different countries are at the edge of completion of the last phase of clinical trials among infants (below 12 years of age) [33].

Vaccination is a cost-effective weapon for the prevention of life-threatening diseases. A vaccine is the most required weapon needed in the present time to fight the deadly SARS-CoV-2 virus. The process of developing a safe and effective vaccine that can be approved for human use is long, costly, and tedious. All over the world, there are 5 types of vaccines being designed to provide a successful immunization against covid-19. The main aim of all the vaccines is to create an immunologic memory against the SARS-CoV-2 virus such that in the future when a person is infected with the virus, the immune system will recognize the virus and trigger a response to combat the disease. Before opening up vaccination for children, clinical trials should be completed.

According to Union Health minister of India, the vaccination of children against COVID-19 may start as early as August, 2021 and both Bharat Biotech's Covaxin and Zydus Cadila's DNA vaccines for the 12-18 age group will be urgent consideration. Most of the children are already exposed to the disease but vaccination is necessary for reducing transmission.

Among all the companies manufacturing vaccines, to date only Pfizer has been authorized for the vaccination of children within the age group of 12 and above (2 doses, each of 3 weeks apart). Like adults, kids even have reactions like mild fever, body ache. The appearance of these symptoms shows that the individual is developing immunity in response to the vaccines. In India, DNA vaccines are underdeveloped for 12-18yrs children as there are predictions that the third wave of
Covid-19 caused by the new Delta plus SARS-CoV-2 variant can affect the kids. The rates of vaccination in elder community are associated with a substantial later decline in infections among the individuals aged under 16 years, who are unvaccinated.

\subsection{Medicine}

The controlled usage of Remdesivir has been reported to have a promising result among the adults with shorter recovery time since the end of last year, but the clinical trials among the children have been started on a narrow scale which has also shown positive results [34]. Other prescribed medications include azithromycin, Rotanavir, Lotanavir etc. More clinical trials are necessary for prescribing medications throughout the world.

\subsection{Maternal antibodies}

A recent study in nature review suggests that lactating women who have been vaccinated are transmitting antibodies against COVID19 to their babies [35]. Earlier studies have suggested that the vaccines (mainly mRNA vaccines) could hardly cross through the breastmilk, but the immunoglobulin A (IgA) and immunoglobulin G (IgG) have been reported to transmit through breast milk [36]. But how long a vaccinated mother would produce antibodies so that their children get the protection or how effective the maternal antibodies are against the virus among their babies are still a matter of concern. More case studies are required to give promising data.

\subsection{Home isolation}

The Ministry of Health and Family Welfare has listed several ways and guidelines to identify if children have Covid-19 symptoms and how to manage the condition. Asymptomatic Covid-19 positive Children can be managed at home with home isolation and symptomatic treatment.

\section{AN INTERPRETATION TO THE THIRD WAVE:WHETHER CHILDREN WOULD BE THE ONLY SUFFERER}

Children being super-spreader of the third wave is being a myth. The virus affects individuals at any age but the symptoms vary from different age groups. Since the reports from the first two waves, the children have been asymptomatic or mild to moderately affected whereas the severity of the full-blown disease have been reported mainly at a section elderly people, the children have also 
been tested in less frequency due to the absence of symptoms in a majority of cases so that it is difficult to conclude that they had been less affected by the virus. They can also transmit the virus while being asymptomatic. But as the vaccine drive has been peaked among people above 18 years of age, it is being thought that the children being not vaccinated might be the possible target for the third wave. But according to the case studies from previous experiences, scientists are not expecting it to be much fatal for the young ones.

\section{CONCLUSION}

As the third wave is approaching, it is high time to be concerned about the disease. Already the vaccination has started to peak with an urgent need of the nation to survive. Though there is no point to get panic, there is not at all any point to be relaxed. If we can be concerned enough and maintain the protections, we would be able to put evolutionary pressure on the virus to limit its host with compromised virulence. Scientists are expecting the third wave would not be as much as life-threatening as the vaccine drive is already in motion and it would not be supposed to last long like the first two waves, still, the high transmissibility of the delta plusstrain of the virus cannot be ignored. And most importantly there is not sufficient data about the efficacy of the vaccine against delta plus. The true burden of the pandemic in children is yet to be unveiled. Needless to say, more case studies are required to confirm the efficacy rate of the vaccine against the delta plus strain.

\section{ACKNOWLEDGEMENT}

We are privileged to convey our sincere gratitude to Prof. Subir Chandra Das Gupta, Head of the Department, Post Graduate Department of Zoology, Maulana Azad College, Kolkata for providing laboratory facilities and financial support under DBT Star College Scheme to conduct the work.

\section{REFERENCES}

1. World Health Organization. Report of the WHOChina Joint Mission on Coronavirus Disease 2019 (COVID-19), 16-24 February 2020, World Health Organization.

2. Nickbakhsh S, Ho A, Marques DFP, McMenamin J, et al. J. Inf. Dis., 2020; 222(1):7-25.
3. Source: PTI Edited By: Utkarsh Mishra: Second wave of Covid not over yet, can't lower guard: says Government. The Hindu, July 2021.

4. Panda PK, Sharawat IK. Ind. J. Ped., 2020; 87(7):556-557.

5. Ludvigsson JF. Acta Ped., 2021;110:914-921.

6. Brodin P. Acta Ped., 2020; 109(6):1082-1083.

7. Hasan M, Ahmad M, Dargham S, Ibrahim H, et al. Res. Squ., 2021; 2:1-11.

8. Dessie G, Malik T. Inf. Drug. Res., 2021; 14:18831892.

9. Jin Y, Yang H, Ji W, Wu W, Chen S, et al. Viruses, 2020; 12(4):372.

10. Toubiana J, Poirault C, Corsia A, Bajolle F, et al. British Medical Journal, 2020; 369:m2094.

11. Hasan G, Sharma A. Curr. Opi. Physiol., 2020; 17:18.

12. Korber B, Fischer WM, Gnanakaran S, Yoon H, et al. Cell, 2020; 182(4):812-827.

13. BanerjeeS, Guha A, Das A, Nandi M, Mondal, R. Ind. Ped., 2020; 57:963-964.

14. Gupta A, Madhavan MV, Sehgal K, Nair N, et al.Nat. Med., 2020; 26(7):1017-1032.

15. https://www.thehindubusinessline.com > article34122816: Covid-19:Children with adrenal insufficiency at 10 times, March 21, 2021.

16. WHO. Corona virus disease (COVID-19) Pandemic,

17. https://www.who.int/emergencies/diseases/nove l-coronavirus-2019> (2020).

18. Rallof J. Science, 2006; 170:312.

19. Liu PT, Stenger S, Li H, Wenzel L, et al. Science, 2006; 311:1770-1773.

20. Marlais M, Wlodkowski T, Vivarelli M, Pape L, et al. Lancet Child Adolescent Health, 2020; 4(7):17-18.

21. Turner D, Huang Y, Martín-de-Carpi J, Aloi M, et al. J. Ped. Gastro. Nutri., 2020; 70(6):727-733.

22. Hoffmann M, Kleine-Weber H, Schroeder S, Kruger N, et al. Cell. 2020; 181(2):271-280.

23. Shang J, Wan Y, Luo C, Ye G, Geng Q, Auerbach A, et al. Proc. Nat.1 Acad. Sci., 2020;117(21):11727.

24. Deng Q, Rasool RU, Russell RM, Natesan R, Asangani IA. Science, 2021; 24(3):102254.

25. Shirato K, Kawase M, Matsuyama S. Virology, 2018; 517:9-15.

26. Hou Y, Zhao J, Martin W, Kallianpur A, et al. BMC Medicine, 2020; 18(1):216.

27. Cattrini C, Bersanelli M, Latocca MM, Conte B, et al. Cancers, 2020; 12(8):2325. 
28. Bennani NN, Bennani-Baiti IM. Ann. Onco., 2020; 31(11):1585-1586.

29. Schuler BA, Habermann AC, Plosa EJ, Taylor CJ, et al. bioRxiv, 2020.

30. Callaway E. Nature, 2021; 595:346-348.

31. COVID-19 Host Genetics Initiative. Nature, 2021.

32. Nakanishi T, Pigazzini S, Degenhardt F, Cordioli M, et al., medRxiv, 2021.

33. Bhatia R. Curr. Opin. Psych., 2020; 33(6):568-570.
34. Rajapakse N, Dixit D. Ped. Int. Ch. Health., 2020; 41(1):36-55.

35. Méndez-Echevarría A, Pérez-Martínez A, Gonzalez Del Valle L, Ara MF, et al. Eur.J.Ped., 2021; 180(4):1317-1322.

36. Hall BS. Nature, 2021; 594:492-494.

37. Friedman MR, Kigel A, Bahar Y, Yogev Y, Dror Y, Lubetzky R, Wine Y. medRxiv, 2021; 2003. 2006. 21252603. 